\title{
La adaptación de los animales al medio. Qué aspectos consideran los estudiantes de Primaria y Secundaria
}

\author{
Susana García-Barros (D), $\mathbf{M}^{\mathrm{a}}$ Jesús Fuentes Silveira (D), Juan-Carlos Rivadulla-López (D), \\ Lucía Vázquez-Ben (iD) \\ Departamento de Pedagoxía e Didáctica. Facultade de Ciencias da Educación. Universidade da Coruña. \\ España.susana.gbarros@udc.es,m.j.fuentes@udc.es,juan.rivadulla@udc.es,lucia.vben@udc.es
}

[Recibido: 15 febrero 2021. Revisado: 18 marzo 2021. Aceptado: 6 junio 2021]

\begin{abstract}
Resumen: La adaptación es una característica esencial de los seres vivos cuyo estudio contribuye a conocer el medio en el que viven, pero también a explicarlo, pues constituyen una idea clave para la construcción de un modelo tan relevante como el de evolución. Por tal motivo en este estudio se analizan las ideas adaptativas que activan los estudiantes de $5^{\circ}$ de Primaria y de $1^{\circ}$ de ESO cuando diseñan un animal imaginario que habita el desierto. Los resultados muestran que los participantes describen sin problema un prototipo de desierto y centran las adaptaciones del animal diseñado en las necesidades primarias: obtención de agua/alimento y defensa de otros seres vivos. Las adaptaciones se asocian a rasgos morfológicos, seguidos de los fisiológicos y de comportamiento del animal. Finalmente se reflexiona sobre la importancia didáctica de este tipo de adaptaciones.
\end{abstract}

Palabras clave: educación primaria; educación secundaria; adaptación; animales; ideas de los alumnos.

The adaptation of animals to the environment. What aspects do Primary and Secondary students consider?

\begin{abstract}
Adaptation is an essential characteristic of living beings. Its study contributes to knowing the environment in which they live, but also to explaining it, since it constitutes a key idea for the construction of a relevant model like evolution. For this reason, this study analyzes the adaptive ideas that students in 5th Primary Education and 1st ESO activate when they design an imaginary animal that lives in the desert. The results show that the participants describe a desert prototype without problem and focus the adaptations of the designed animal on the primary needs: obtaining water/food, and defense of other living beings. The adaptations are associated with morphological traits, followed by physiological and animal behavior. Finally, it's reflected on the didactic importance of this type of adaptations.
\end{abstract}

Keywords: primary education; secondary education; adaptation; animals; student ideas.

Para citar este artículo: García-Barros S., Fuentes Silveira M. J., Rivadulla-López J. C. y Vázquez-Ben L. (2021) La adaptación de los animales al medio. Qué aspectos consideran los estudiantes de Primaria y Secundaria. Revista Eureka sobre Enseñanza y Divulgación de las Ciencias 18(3), $3106 . \quad$ doi: 10.25267/Rev_Eureka_ensen_divulg_cienc.2021.v18.i3.3106

\section{Introducción}

Desde hace ya años, existe un amplio consenso respecto a que la enseñanza de las ciencias debe promover la alfabetización científica de los ciudadanos, lo que le permitirá conocer y explicar el funcionamiento del mundo que nos rodea (NRC 2007) e intervenir adecuadamente en la sociedad tomando decisiones responsables (Pujol 2003; COSCE 2011). Lo indicado resulta coherente con el desarrollo de la competencia científica (Sanmartí y Márquez 2017). Desde esta perspectiva el estudio de los seres vivos se percibe como un núcleo temático a considerar ya desde los primeros niveles educativos. Con él se pretende que los estudiantes vayan incrementando sus experiencias, respeto y conocimientos al tiempo que desarrollan un modelo de ser vivo cada vez más elaborado. Para este desarrollo es imprescindible tomar en consideración la interacción de los organismos entre sí y con su medio, así como su evolución y cambio (Cañal 2008; Caravita y Falchetti 2005; Pujol 2003), aspectos coherentes con los enfoques evolutivo y ecológico que sirven de orientación a la organización del contenido biológico (Bermúdez y Occelli 2020). En este marco la adaptación, incluso en el sentido más sencillo centrado en la identificación de las características que favorecen la supervivencia de 
los seres vivos en un determinado medio, constituye una idea relevante para percibir la diversidad, constructo imprescindible para construir el modelo evolutivo en el marco del ecosistema (Lehrer y Schauble 2012).

Basándonos en lo indicado, e independientemente de la controversia que pueda existir en relación a cuando debe introducirse el tratamiento de la evolución en la Educación Primaria (EP) (Vázquez-Ben y Bugallo-Rodríguez 2018), la adaptación debería contemplarse ya en este nivel (Borgerding y Kaya 2019) y debería tratarse más adecuadamente en los libros de texto de la educación obligatoria para facilitar los aprendizajes que este constructo potencia (De la Gándara, Gil-Quílez y Sanmartí 2002; Prinou, Halkia y Skordoulis 2011). Sin embargo, a pesar de su importancia la adaptación es poco considerada en el currículum oficial LOMCE (Comunidad autónoma de Galicia), pues está ausente en EP y su presencia resulta limitada en ESO, al vincularse en $1^{\circ}$ curso solo al bloque de los seres vivos -biodiversidad en el planetasin referirse a ella en el bloque de los ecosistemas. Por otra parte, la investigación dirigida a conocer en qué medida los estudiantes son capaces de identificar adaptaciones en los seres vivos tampoco ha sido extensa, por ello en este trabajo intentamos abordar específicamente este problema con estudiantes de Primaria y ESO.

\section{Marco teórico}

\section{El modelo escolar de ser vivo}

Aunque en la enseñanza de las ciencias la idea de modelo y de modelización puede tener distintas acepciones (Oliva 2019), la enseñanza-aprendizaje basada en modelos se percibe como un marco teórico que puede dirigir la progresión de aprendizajes necesarios para explicar el mundo. Tal progresión va desde los modelos mentales del alumno a aquellos más elaborados adaptados a las distintas edades, que define la ciencia escolar (Clement 2000). Los modelos escolares integran una serie de conceptos y entidades bien organizados (Cañal 2004), que se pueden ir ampliando de tal forma que en el proceso de modelización el alumno puede transitar a través de ellos (Acher 2014), favoreciéndose así la práctica científica auténtica. En este sentido, la selección de grandes ideas resulta trascendente (Harlen 2013), pues en relación a ellas se ponen en juego las habilidades y capacidades necesarias para la comprensión del mundo físico y de los seres vivos y, en definitiva, para el desarrollo de la competencia científica. Así, los modelos escolares sirven tanto al diseño del currículum, como al profesorado para tomar decisiones sobre la propia progresión, o la secuenciación de actividades de aula (Oliva 2019).

El modelo escolar de ser vivo, al igual que otros modelos de la ciencia escolar, toma como referente el modelo científico, sin embargo, definir la vida no es sencillo y los propios biólogos así lo reconocen (Garbisu, Amézaga, Albizu, y Alkorta 2003), quizás porque resulta excesivamente filosófico. Desde una perspectiva científica se han propuesto distintas teorías que explican lo que es la vida y algunas han tenido influencia en la construcción del modelo escolar. Una de ellas es la autopoiesis (Maturana y Varela 1980) que postula que los organismos vivos son sistemas especialmente organizados que mantienen dicha organización gracias a un continuo intercambio de materia con el medio, y otra es la teoría sintética de la evolución. Desde esta última se señala que los seres vivos se perpetúan transmitiendo características genéticas que son mutables, siendo la selección natural la que provoca la perpetuación de los mejor adaptados a un determinado medio que también está sometido a cambios. Desde este marco teórico y tomando como referente las aportaciones de distintos autores (Cañal, 2008, Pujol 2003; Garrido y Martínez Losada 2009, Martínez Losada, García Barros, Garrido 2014) podemos resumir que el modelo escolar de ser vivo debería atender a las siguientes ideas clave: a) Los seres vivos necesitan obtener materia/energía para mantener 
una permanente reconstrucción de ellos mismos, para ello interaccionan constantemente con el medio y con otros seres vivos a través de continuos procesos, originando modificaciones en el medio; b) Los seres vivos se perpetúan, producen copias similares de sí mismos, raramente idénticas, que sobreviven siempre y cuando sus características sean idóneas para mantenerse en un hábitat cambiante. Estas ideas pueden desarrollarse a través de distintos submodelos que van poniendo el foco de la vida a diferentes niveles que transitan entre el moléculo/celular, el organismo y el ecosistema (García Rovira 2005).

\section{La adaptación}

Un concepto destacado en el modelo de ser vivo es la adaptación, pues constituye un eslabón entre las necesidades autopoiéticas de los individuos y el proceso evolutivo que, en definitiva, explican los rasgos adaptativos que satisfacen dichas necesidades. Sin embargo, el concepto de adaptación es controvertido, criticándose la tendencia adaptacionistas que postula que los rasgos adaptativos óptimos son únicamente el resultado de la selección natural (Gould y Lewontin 1979). Un análisis profundo de distintas aportaciones teóricas, ha llevado a Reeve y Sherman (1993) a definir la adaptación como una variante fenotípica que da como resultado la mayor aptitud entre un conjunto específico de variantes en un entorno dado. Los autores admiten que la teoría de la selección natural predice que el fenotipo más adaptado será el predominante, pero pueden entrar en juego otros procesos selectivos como la deriva, la migración o la selección que actúa sobre un rasgo correlacionado. En este mismo sentido también se pronunciaron otros (Moreno 2006).

Independientemente de estas discusiones, desde la didáctica de las ciencias la adaptación ha estado vinculada a la evolución, línea de investigación pujante, desde la que se siguen estudiando las ideas de los alumnos (Larrain, López, Gómez, Verdugo, Freire, Grau, Cosmelli y Bonhomme 2021) y su capacidad para transferir conocimientos evolutivos a diversas situaciones (Kevin, Pugh, Kristin, Koskey y Linnenbrink-Garcia 2014), o desde la que se reivindica la actualización de su tratamiento didáctico, incorporando otros factores causales del cambio evolutivo, además de la selección natural (Araujo 2020). Concretamente la adaptación se ha asociado a una de las grandes ideas, la ecología, que junto a la variedad y al cambio, fueron consideradas clave en la elaboración de una estructura de progresión dirigida a la construcción del modelo de evolución a lo largo del sistema educativo (Lehrer y Schauble 2012). Aquí el ecosistema se contempla como el escenario en el que los más pequeños pueden ir apreciando los rasgos adaptativos de los seres vivos en distintos ambientes, llegando a considerar la relación coste-beneficio de los mismos para su supervivencia. El conocimiento más elaborado se conseguirá cuando ya se admita la variación ambiental y su implicación en la reproducción y en la supervivencia. Por otra parte, la adaptación se toma como pregunta mediadora, - ¿qué proceso es responsable de la adaptación biológica? - en una propuesta de enseñanza para la construcción del modelo de evolución en secundaria (Pérez, Gómez Galindo y González Galli 2018). Así mismo, emerge en el desarrollo de otra propuesta orientada a la introducción de conceptos evolutivos incluso a niños pequeños (4-6 años). En ella, mediante el uso de narraciones y el modelado de un animal ficticio con arcilla, los niños fueron capaces de diseñar su animal y justificar la utilidad de sus rasgos para sobrevivir en diferentes hábitats, relacionando estos, sobre todo, con la obtención de alimentos (Frejd, Stolpe, Hultén y Schönborn 2020).

La adaptación ha sido tratada como núcleo de estudio específico en otros trabajos, concretamente en la construcción fundamentada del modelo de ser vivo con niños de primaria solicitándose el diseño de animales del desierto y la justificación de sus características adaptativas (Gil Quílez, de La Gándara, Dies Álvarez y Martínez Peña 2011). En esta misma línea, Horton y Friedenstab (2013) propusieron que los niños predijeran qué animal, de una 
serie de ejemplares, estaría mejor adaptado a distintos tipos de desierto. En otro planteamiento se emplearon analogías con objetos cotidianos, apreciándose que el recurso favorecía que los niños de primaria establecieran relaciones forma-función (Rule, Baldwin y Schell 2008). También se evidenció la capacidad que tienen los niños de 3-6 años, asistentes a un campamento de ciencias, para situar justificadamente ciertos seres vivos en determinados ambientes (bosque caducifolio, zona polar, desierto) (Borgerding y Kaya 2019). En este caso, incluso los niños más pequeños establecían buenas relaciones organismos-biomas, aunque la adecuación de las justificaciones aportadas dependía de la edad. Solo algunos de los mayores, centraban sus razones en rasgos adaptativos específicos del organismo, o en la identificación de la variedad «polar» del mismo, mientras que los pequeños daban más razones egocéntricas, basadas en sus gustos.

Sobre adaptación además se publicaron otras propuestas que adoptan un diseño más concreto, focalizando específicamente en el medio físico, específicamente en la adaptación del mantenimiento de la temperatura corporal, tomando la piel el papel de aislante (Rowlands, Hokayem y Biediger 2017). La importancia adaptativa de la piel también sirvió en otra propuesta para estudiar la transmisión de las características a los descendientes, destacando la diversidad de pelajes dentro de una misma población (Royce 2019). Los rasgos adaptativos también sirvieron para mostrar características específicas que implican una relación con otros seres vivos, concretamente las adaptaciones de la vista que permiten al animal comunicarse, obtener comida, encontrar pareja o evitar depredadores (Royce 2017).

\section{La adaptación en el currículum de Educación Primaria y ESO}

La adaptación tiene escasa presencia en el currículum LOMCE (Comunidad autónoma de Galicia), concretamente en EP no se menciona en contenidos, ni en criterios de evaluación, ni en estándares en ningún curso. En ESO se contempla de forma reduccionista, pues se pone el foco en el ámbito descriptivo, siendo anecdótica su dimensión explicativa que la relaciona con el modelo evolutivo y la selección natural. Así en $1^{\circ}$ de ESO, y solo en el bloque «La biodiversidad en el planeta Tierra», se encuentran contenidos como, «... adaptaciones de los animales y plantas al medio» y en $4^{\circ}$ se consideran «los factores limitantes y adaptaciones» en el bloque «Ecología y medio ambiente», siendo la relación de la adaptación con la evolución anecdótica, citada en un estándar -«Establece la relación entre variabilidad genética, adaptación y selección natural» del bloque «La Evolución de la vida»-, constituyendo una muestra más de la desconexión entre ecología y evolución.

\section{Objetivo}

Conocer en qué medida los alumnos de $5^{\circ}$ curso de EP y de $1^{\circ}$ de ESO son capaces de describir un medio hostil para la vida, como es el desierto, y diseñar un animal adaptado al mismo. Se trata de averiguar:

1. Qué características concretas activan en la descripción del medio.

2. En términos generales, hacia qué factores del medio dirigen la adaptación del animal diseñado y en qué rasgos del mismo focalizan las adaptaciones.

3. Qué relaciones específicas adaptación-características del animal resultan más frecuentes en el animal diseñado.

\section{Metodología}

Se diseñó una propuesta de actividades para los últimos cursos de EP y a $1^{\circ}$ de ESO dirigida al estudio de la adaptación de los animales. La propuesta comienza con unas preguntas iniciales para deliberar sobre qué necesitan los animales para vivir y sobre la adaptación a diversos 
hábitats de especies conocidas (águilas, merluzas, moscas...). Después de esta deliberación el alumnado deberá pensar en un hábitat concreto, el desierto, e inventar un animal adaptado al mismo, contestando a estas cuestiones:

1. Piensa ahora en las características de un desierto, fijate en: cómo es el paisaje, el suelo, si hay seres vivos, etc. Descríbelo, puedes hacer un dibujo.

2. Inventa un animal que pueda vivir en él. Tienes que pensar que debe estar adaptado, es decir debe poder conseguir comida, conseguir y conservar agua... En definitiva, se trata de que pongas sus características y que las relaciones con los beneficios que les aportan para vivir en ese medio. Haz el dibujo de tu animal. Aquí se incluye una tabla con dos columnas para facilitar la relación: características/beneficios.

La actividad se implementó en $5^{\circ} \mathrm{EP}$ y en $1^{\circ}$ de ESO, previa presentación a los profesores colaboradores, con los que se discutió la intención y adaptación de la misma. Se propuso que su realización fuera después que los alumnos hubieran estudiado los seres vivos y sus características. Concretamente se integraron las actividades en el marco del estudio del ecosistema. Cabe indicar que el contexto de ejecución fue diferente en ambos niveles educativos pues, en EP se realizó en el aula bajo la presentación/dirección de la maestra que supervisaba la tarea, interviniendo solo para resolver dudas técnicas; en ESO, debido al confinamiento, se realizó de forma telemática. El alumnado recibió la actividad y su explicación que hacía hincapié en la importancia de reflexionar sobre las cuestiones iniciales (necesidades vitales de animales y la adaptación de ejemplares conocidos).

Los participantes fueron: 80 estudiantes (44 niñas y 36 niños) de $5^{\circ}$ de EP de 2 centros, y 58 (28 chicas y 30 chicos) de 3 centros de ESO. Todos ellos pertenecientes a la provincia de A Coruña.

Las producciones individuales de los estudiantes se analizaron empleando dosieres, considerando categorías y subcategorías cada vez más específicas. Para analizar las descripciones del desierto y las adaptaciones del animal se tomaron como referente los factores bióticos y abióticos del ecosistema, contemplándose también otras categorías de carácter empírico. Los rasgos adaptativos se vincularon a tres categorías: morfología, fisiología y comportamiento (tabla 1). Los dibujos fueron utilizados como complemento de las producciones escritas (ejemplos en Anexo).

En el análisis de los prototipos se combinó el tipo de adaptación con el rasgo adaptativo del animal, pues una misma adaptación puede asociarse a varias características del animal y un tipo de características a distintos tipos de adaptaciones.

Además de los análisis anteriormente citados, se establecieron niveles de mayor a menor adecuación de los prototipos:

- Nivel 1. Contemplan al menos adaptaciones a 2 factores abióticos y 2 bióticos del medio. Asocian las adaptaciones a 2 o 3 tipos de características del animal (anatomía/fisiología/ comportamiento).

- Nivel 2. Contemplan al menos adaptación a un factor biótico y a uno abiótico. Asocian las adaptaciones a 2 o 3 tipos de características.

- Nivel 3. Contemplan al menos adaptación a un factor bióticos y a uno abióticos. Las adaptaciones se asocian a un solo tipos de características.

- Nivel 4. Contemplan un solo tipo de adaptaciones bióticas o abióticas que se asocia a 2 o 3 características.

- Nivel 5. Se contemplan un solo tipo de adaptación bióticas o abióticas que se asocian a un solo tipo de características.

- Otros. Se omite adaptación o características 
Tabla 1. Categorías empleadas en el análisis de las producciones del alumnado

\begin{tabular}{|c|c|c|c|}
\hline $\begin{array}{c}\text { ASPECTO } \\
\text { ANALIZADO }\end{array}$ & CATEGORÍAS & & SUBCATEGORÍAS \\
\hline \multirow{9}{*}{$\begin{array}{l}\text { Descripción } \\
\text { del desierto }\end{array}$} & \multirow{4}{*}{ Factores abióticos } & \multicolumn{2}{|l|}{ Agua } \\
\hline & & \multicolumn{2}{|l|}{ Temperatura } \\
\hline & & \multicolumn{2}{|c|}{ Fenómenos atmosféricos (viento/tormentas) } \\
\hline & & \multicolumn{2}{|l|}{ Suelo/paisaje } \\
\hline & \multirow{2}{*}{ Factores bióticos } & \multicolumn{2}{|c|}{ Referencias genéricas a animales/plantas } \\
\hline & & \multicolumn{2}{|l|}{ Especies concretas } \\
\hline & \multirow{2}{*}{ Interacciones } & \multicolumn{2}{|l|}{ Entre seres vivos } \\
\hline & & \multicolumn{2}{|c|}{ Entre seres vivos y medio } \\
\hline & \multicolumn{3}{|c|}{ Otras*. Respuestas antropocéntricas y sociales (construcciones/rebaños/caravanas) } \\
\hline \multirow{20}{*}{$\begin{array}{l}\text { Diseño del } \\
\text { animal } \\
\text { imaginario }\end{array}$} & \multirow{8}{*}{ Adaptaciones } & \multirow{4}{*}{$\begin{array}{l}\text { Factores } \\
\text { abióticos }\end{array}$} & Agua \\
\hline & & & Temperatura \\
\hline & & & Fenómenos atmosféricos (viento/tormentas) \\
\hline & & & Suelo/paisaje \\
\hline & & \multirow{4}{*}{ Factores bióticos } & Defensa frente a otros seres vivos \\
\hline & & & Obtención de alimento \\
\hline & & & Búsqueda de pareja \\
\hline & & & Cuidado de crías \\
\hline & \multirow{12}{*}{$\begin{array}{c}\text { Características a } \\
\text { las que se } \\
\text { vinculan las } \\
\text { adaptaciones }\end{array}$} & \multirow{5}{*}{ Morfología } & Tamaño (grande/pequeño) \\
\hline & & & Piel (color, grosor) \\
\hline & & & Extremidades \\
\hline & & & Órganos de los sentidos \\
\hline & & & Otras estructuras (colas, jorobas...) \\
\hline & & \multirow{3}{*}{ Fisiología } & $\begin{array}{l}\text { Producción de sustancias (veneno, regenera } \\
\text { estructuras...) }\end{array}$ \\
\hline & & & Mantenimiento de temperatura \\
\hline & & & Otras funciones (rapidez, valentía, inteligencia...) \\
\hline & & \multirow{4}{*}{ Comportamiento } & Socialización (pareja, manada, cuidados...) \\
\hline & & & Cobijo (nidos, madrigueras...) \\
\hline & & & Costumbres (nocturnas, diurnas...) \\
\hline & & & $\begin{array}{l}\text { Tipo de alimentación (carnívoro, herbívoro, } \\
\text { omnívoro) }\end{array}$ \\
\hline
\end{tabular}

*Origen empírico

\section{Resultados}

\section{Descripción del desierto}

Los estudiantes describen un desierto prototípico seco y solo uno de ESO, se refiere además al desierto polar. Todos mencionan características físicas (factores abióticos), atendiendo a la temperatura y al suelo/paisaje (alrededor del $90 \%$ y el $84 \%$ ) y en menor proporción a la escasez de agua $(67 \%$ de los sujetos de ambos grupos) o a otros fenómenos atmosféricos (vientos, tormentas, ...), menos detectados en EP que en ESO (figura 1). Respecto a la temperatura prácticamente todos señalan que son altas, matizando la existencia de cambios bruscos $(35 \% \mathrm{EP}$ y $31 \%$ ESO). El suelo y el paisaje fue descrito de forma prototípica (suelo arenoso/pedregoso, dunas, oasis). Además, un pequeño grupo de alumnos (3,75\% de EP y 6,9\% de ESO) sitúa zonas desérticas en África o Australia y/o cita desiertos concretos (Sahara o Gobi).

Todos los estudiantes, excepto dos de ESO, admitieron la existencia de seres vivos (factores bióticos). La mayoría citaron animales y vegetales $(68,8 \% \mathrm{EP}$ y $75,9 \% \mathrm{ESO})$, algunos lo hicieron genéricamente, y otros señalaron especies vegetales, especialmente en EP $(85 \%$ frente a $68,9 \%$ ESO), aunque circunscribiéndose a cactus (68,8\% EP y $51,7 \%$ ESO) y/o palmeras (55\% EP y 20,7 \% ESO). Las referencias a especies animales fueron similares en ambos grupos (EP 57,5\% y ESO 63,8\%), pero más diversas en ESO (30 especies frente a 16 en EP), 
destacando camello/dromedario (52,5\% en EP y 39,7\% en ESO) y escorpión (27,5\% en EP y $18,9 \%$ en ESO). Varios participantes sobre todo de EP incluyeron aspectos humanos (pirámides, caravanas, rebaños...) (36,3\% frente al 22,4\% ESO).

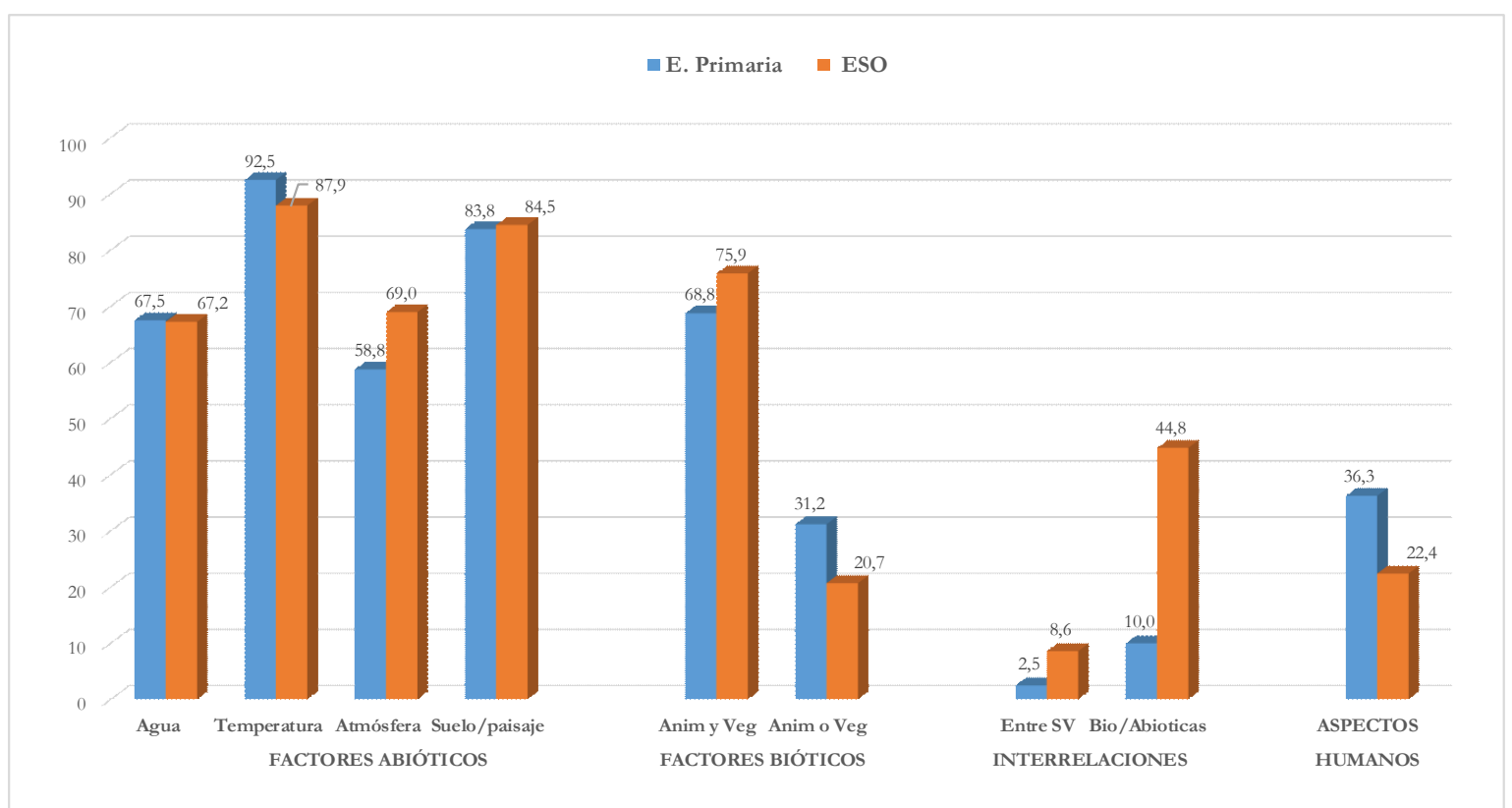

Figura 1. Aspectos considerados en la descripción del desierto por el alumnado

En la descripción del desierto, 7 alumnos (2 de EP y 5 de ESO) mencionaron interrelaciones de los seres vivos, bien entre animales, «hay pocos animales carnívoros porque no hay herbívoros», o entre animales y vegetales, «....suelen tener espinas, corteza dura como medida de protección frente a animales...». Las referencias adaptativas a los factores abióticos fueron más frecuentes, sobre todo en ESO (44,8\% frente al 10\% en EP): «animales capaces de sobrevivir al clima desértico»; «los vegetales que hay no necesitan mucha agua»; «Hay pocos animales debido a que no muchos soportan el calon».

\section{Adaptaciones de animal imaginario}

Los estudiantes dirigieron las adaptaciones del animal, más hacia los factores bióticos del medio (alrededor del 90\%) que hacia los abióticos, apreciándose aquí mayor diferencia entre grupos (figura 2). Además, vincularon las adaptaciones a distintas características de su prototipo, más a las morfológicas que a las fisiológicas y al comportamiento, especialmente en EP (figura 3).

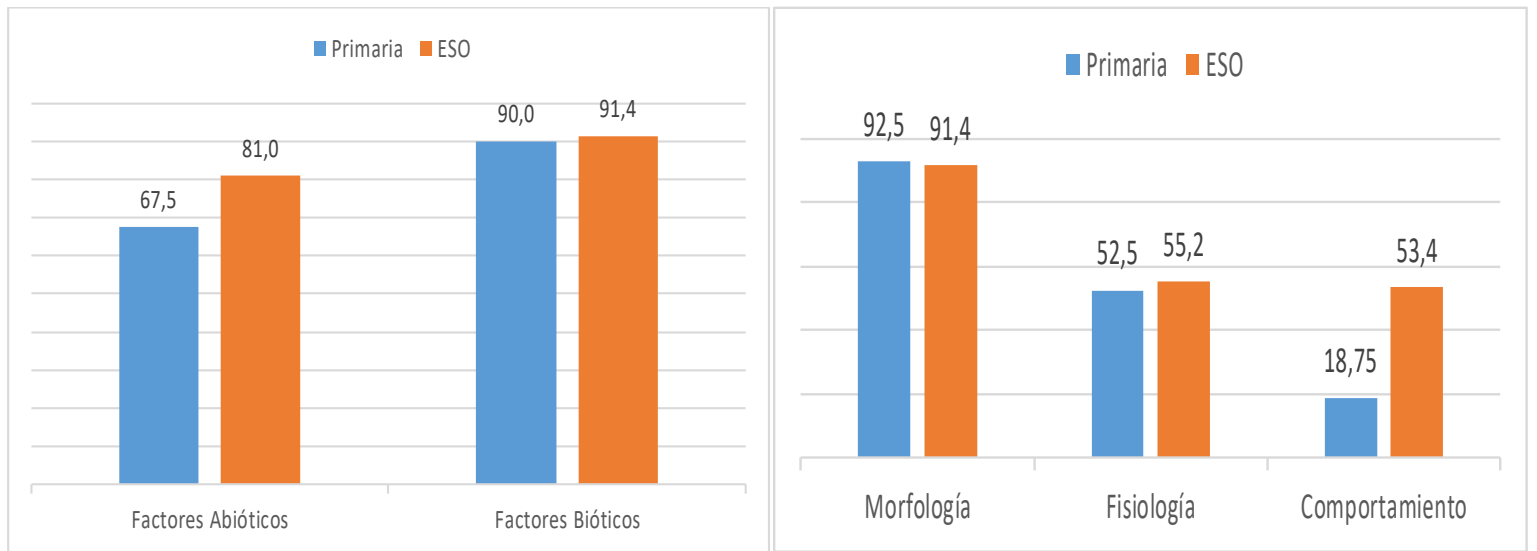

Figura 2. Porcentaje de alumnos que asocian las adaptaciones a aspectos bióticos o abióticos del medio.
Figura 3. Porcentaje de alumnos que asocian adaptaciones a los tipos de características del animal. 
En la figura 4 se concretan las adaptaciones a factores bióticos y abióticos consideradas por el alumnado. Respecto a las segundas, y en ambos grupos, la adaptación a la escasez de agua fue la más señalada (47,5\% EP y 63,8\% ESO), seguida de la dirigida a las altas temperaturas y sus cambios, al tipo de suelo y por último a las inclemencias atmosféricas (viento/tormentas). Las frecuencias fueron superiores en ESO que en EP, especialmente respecto a la temperatura.

Las adaptaciones bióticas más frecuentes se refirieron a la protección frente a otros seres vivos y a la obtención de alimento (más del 60\% de ambos grupos), siendo minoritarias las relacionadas con la reproducción, como conseguir pareja o cuidar las crías, más considerada en ESO (25,9\%).

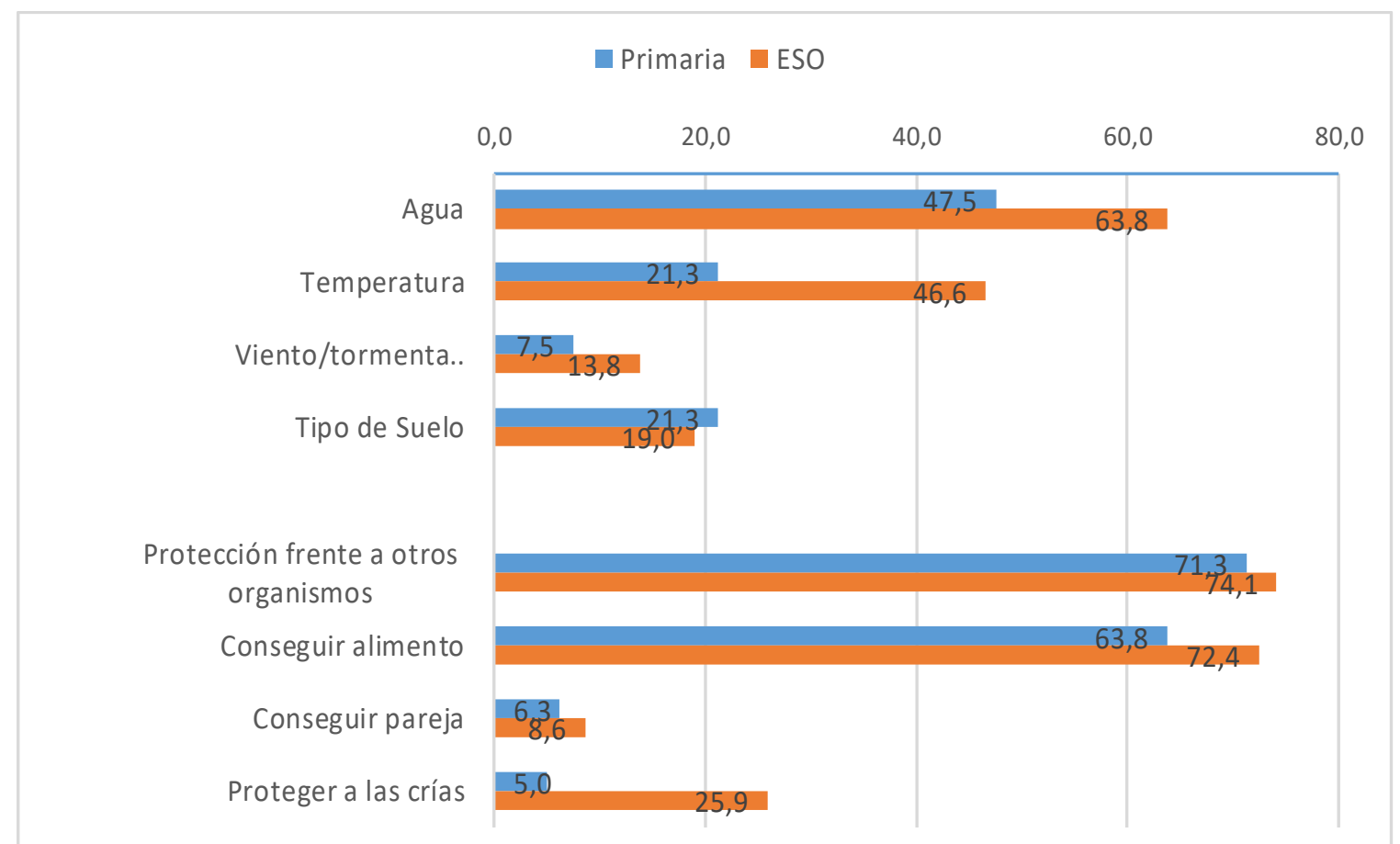

Figura 4. Aspectos concretos a los que los alumnos vinculan las adaptaciones del animal diseñado (expresado en $\%$ de alumnos).

En la figura 5 se recogen las características específicas del animal a las que los estudiantes asocian las adaptaciones. Respecto a las morfológicas, entre el $48 \%$ y el $62 \%$ de los sujetos de ambos grupos lo hacen con las extremidades, la piel, u otras estructuras (depósitos, aguijones, etc.). También lo hicieron, aunque en menor medida, con el tamaño del animal o con los órganos de los sentidos, esto último más en ESO. Concretamente, respecto al tamaño, las alusiones a animal grande o pequeño fueron similares en ESO, predominando la referencia grande en EP (15 alumnos frente a 2 que asocia la adaptación al pequeño tamaño). Respecto a la piel las adaptaciones se relacionaron con el color y/o con el tipo (escamas, escudos, púas, capas, ...), en ESO fueron más frecuentes las referidas al tipo que al color $(55 \%$ y $17 \%)$ y en EP fueron equilibradas. Las adaptaciones vinculadas a los órganos de los sentidos se centraron más en ojos/vista y orejas/oído que en olfato/gusto, con una presencia baja especialmente en EP (5\%).

Las adaptaciones fisiológicas se centraron en la producción de sustancias/venenos, y en menor medida en el mantenimiento la temperatura, siendo más abundantes otras adaptaciones como agilidad, rapidez, inmunidad, etc., (37,5\% en EP y 43,1\% en ESO).

Las adaptaciones relacionadas con el comportamiento se refieren: a las ventajas o no de la socialización (vivir solo, en pareja, manada, ...); a la disposición de cobijo; a las costumbres (nocturnas, diurnas); al tipo de alimentación (herbívoro, omnívoro...). Cada uno de estos aspectos se identificaron entre el 20\% y el 30\% de los diseños de los alumnos de ESO, siendo minoritarios en EP. 


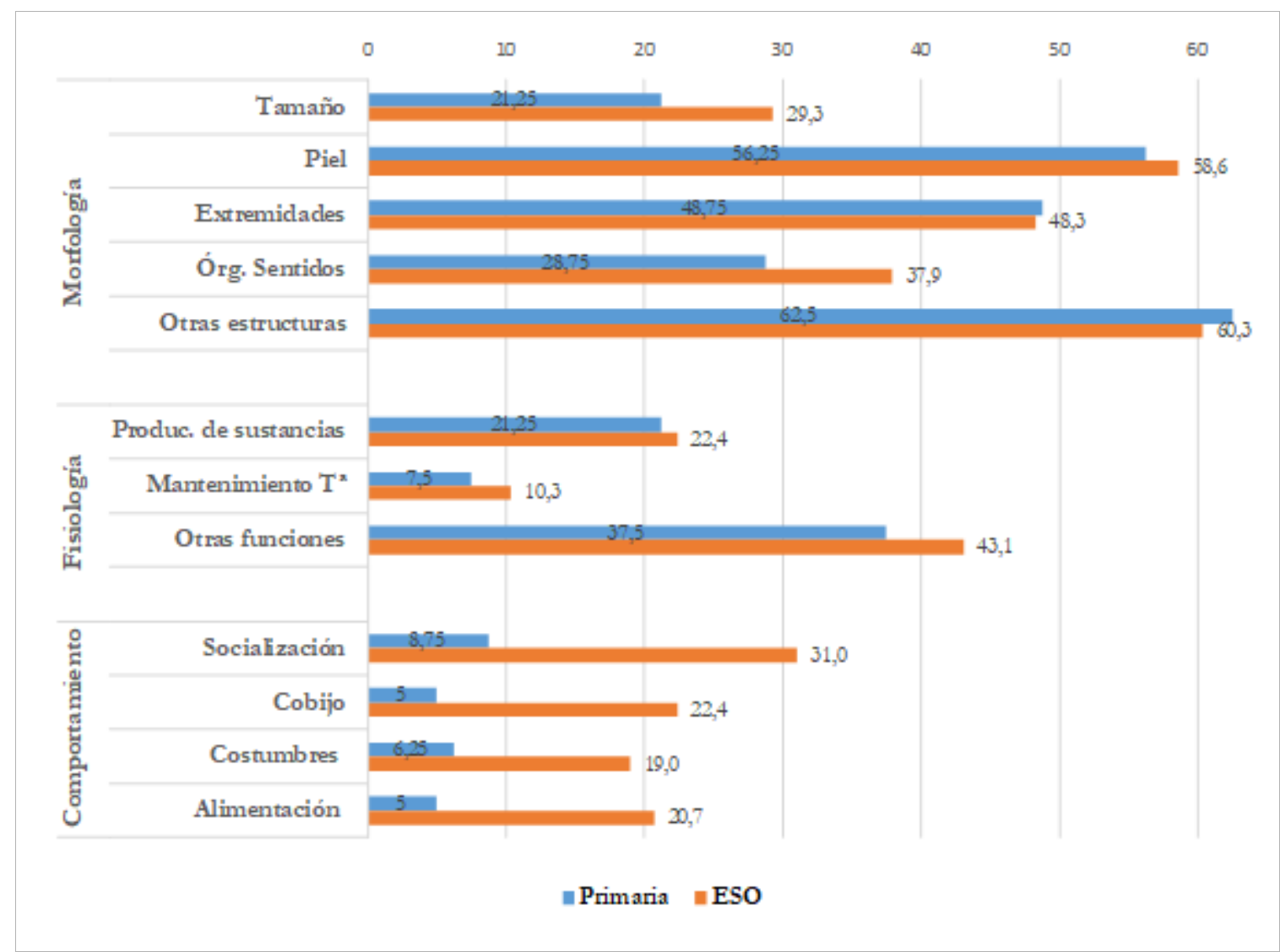

Figura 5. Características específicas del animal a las que los alumnos vinculan las adaptaciones (expresado en \% de alumnos).

En la tabla 2 se muestran las relaciones entre adaptación y rasgos adaptativos del animal diseñado. Destacamos a continuación las más representativas:

a. La escasez de agua se relacionó con todas las características morfológicas y fisiológicas consideradas, y con algunas de comportamiento, todas ellas tendentes a conseguir, conservar o economizar agua. Las relaciones más frecuentes se establecieron con: a) otras estructuras morfológicas «disponer de jorobas, bolsas... para almacenar agua» o «...una lengua, trompa... para beber en sitios inaccesibles» y b) otros aspectos fisiológicos, «necesita beber poco», «respira muy lentamente para disminuir la pérdida de agua».

b. La adaptación a la temperatura se vinculó en ESO a las tres categorías, y en EP a dos (morfología y fisiología). Las relaciones más frecuentes en ambos niveles se establecieron con: a) las características de la piel, como su color, «claro para que no se queme», o su tipo, como el grosor «para soportar calor/frío» o la delgadez/inexistencia «no tiene piel para soportar el calon»; b) los órganos de los sentidos, «orejas grandes con capilares finos para regular su temperatura»; c) el comportamiento, específicamente con el cobijo, «vive en grandes madrigueras para protegerse de las temperaturas», y con las costumbres «hábitos nocturnos para no aguantar el calor por el día».

c. La adaptación a fenómenos atmosféricos (vientos) y al suelo se relacionaron con la morfología. Los rasgos más frecuentes fueron extremidades adaptadas al suelo, «tiene patas cortas, le ayuda a desplazarse mejor», "patas fuertes. Aguanta su peso en un terreno inestable».

d. La protección/defensa frente a otros seres vivos, fue vinculada, prácticamente, a todas las características del animal. Los rasgos adaptativos más frecuentes fueron: a) la piel, «es de color arena para camuflarse», «tiene concha. Se protege de los depredadores»; b) el tamaño, «tamaño XXL; para intimidar a los depredadores»; d) las extremidades, «tiene 
unas patas con forma de pala para enterrarse en el suelo. Puede esconderse de los depredadores...»; e) los órganos de los sentidos, «orejas de conejo para detectar el peligro»; f) la producción de sustancias/ruido, «tiene cola de mofeta para echarle pedos a su rival», «tiene una cola roja y azul que hace un ruido molesto, así escapan los depredadores»; g) otras capacidades fisiológicas, «es muy rápido; no se le puede pillan», «es valiente, se defiende de animales muy grandes»; h) la socialización, «vive en manada para defenderse», o es solitario «..., así tiene menos posibilidades de ser atrapado».

e. La adaptación a la obtención de alimento, se asoció a diversos rasgos, siendo los más frecuentes: a) otras estructuras morfológicas, «tiene un lazo en la cola que le permite atacar a sus presas»; «tiene un cuello muy largo para llegar a las ramas de los árboles y alimentarse»; b) las extremidades, «tiene pies grandes para aplastar a sus presas»; c) la socialización, en grupo «viven en manadas para conseguir más comida» o solitario «..., así no compite con otros animales de su especie por el alimento»; d) costumbres alimenticias, «comen casi cualquier cosa» «comen de todo para no pasar hambre nunca».

f. Las adaptaciones respecto a la reproducción (búsqueda de pareja y/o cuidado de crías) se asociaron básicamente al comportamiento -socialización-, «se reúne solo para tener crías, las cuida después la hembra»; "Cuida a sus crías los primeros meses de su vida».

Respecto a los niveles de adecuación de los prototipos diseñados por los estudiantes se aprecia que más del 65\% de los de ESO se corresponden con los más adecuados (nivel 1 o 2), aunque este porcentaje desciende a menos del 39\% en EP, donde el nivel 5, menos adecuado, tiene bastante presencia (figura 6).

Tabla 2. Alumnos que asocian adaptaciones bióticas y abióticas concretas a las características específicas del animal diseñado.

\begin{tabular}{|c|c|c|c|c|c|c|c|c|c|}
\hline \multirow{2}{*}{\multicolumn{2}{|c|}{$\begin{array}{c}\text { Características del } \\
\text { animal }\end{array}$}} & \multirow{3}{*}{$\begin{array}{c}\text { Nivel } \\
\text { EP }\end{array}$} & \multicolumn{4}{|c|}{ Adaptación a factores abióticos } & \multicolumn{3}{|c|}{ Adaptación a factores bióticos } \\
\hline & & & \multirow{2}{*}{$\begin{array}{c}\text { Agua } \\
2(2,5 \%)\end{array}$} & \multirow{2}{*}{$\begin{array}{l}\text { Tempera- } \\
\text { tura }\end{array}$} & \multirow[t]{2}{*}{ Viento } & \multirow{2}{*}{$\begin{array}{c}\text { Suelo } \\
1(1,25 \%)\end{array}$} & \multirow{2}{*}{$\begin{array}{c}\begin{array}{c}\text { Protección/ } \\
\text { Defensa }\end{array} \\
10(12 \%)\end{array}$} & \multirow{2}{*}{$\begin{array}{c}\begin{array}{c}\text { Conseguir } \\
\text { alimento }\end{array} \\
5(6,25 \%)\end{array}$} & \multirow{2}{*}{$\begin{array}{l}\text { Reproduc- } \\
\text { ción } \\
\text { cuidar } \\
\text { crías }\end{array}$} \\
\hline \multirow{10}{*}{ 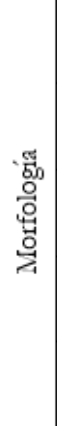 } & \multirow{2}{*}{ Tamaño } & & & & & & & & \\
\hline & & ESO & $2(3,4 \%)$ & $1(1,7 \%)$ & & $1(1,7 \%)$ & $11(19 \%)$ & $2(3,4 \%)$ & \\
\hline & \multirow{2}{*}{ Piel } & EP & $6(7,5 \%)$ & $12(15 \%)$ & $1(1,25 \%)$ & $1(1,25 \%)$ & $26(32,5 \%)$ & $3(3,8 \%)$ & $2(2,5 \%)$ \\
\hline & & ESO & $3(5,2 \%)$ & $15(25,9 \%)$ & $3(5,2 \%)$ & $3(5,2 \%)$ & $20(34,5 \%)$ & $1(1,7 \%)$ & \\
\hline & \multirow{2}{*}{ Extremidades } & EP & $3(3,8 \%)$ & $1(1,25 \%)$ & $1(1,25 \%)$ & $7(8,75 \%)$ & $18(22,5 \%)$ & $16(20 \%)$ & $1(1,3 \%)$ \\
\hline & & ESO & $5(8,6 \%)$ & $4(6,9 \%)$ & $3(5,2 \%)$ & $6(10,3 \%)$ & $9(15,5 \%)$ & $5(8,6 \%)$ & $1(1,7 \%)$ \\
\hline & \multirow{2}{*}{$\begin{array}{l}\text { Órganos } \\
\text { sentidos }\end{array}$} & EP & $1(1,3 \%)$ & & & $4(5 \%)$ & $10(12,5 \%)$ & $11(13,75 \%)$ & \\
\hline & & ESO & $3(5,2 \%)$ & $6(10,3 \%)$ & $1(1,7 \%)$ & & $9(15,5 \%)$ & $5(8,6 \%)$ & \\
\hline & \multirow{2}{*}{$\begin{array}{c}\text { Otras } \\
\text { estructuras }\end{array}$} & EP & $19(23,8 \%)$ & $2(2,5 \%)$ & $2(2,5 \%)$ & $7(8,75 \%)$ & $18(22,5 \%)$ & $23(28,75 \%)$ & $1(1,3 \%)$ \\
\hline & & ESO & $19(32,8 \%)$ & $4(6,9 \%)$ & $1(1,7 \%)$ & & $7(12,1 \%)$ & $22(37,9 \%)$ & $3(5,2 \%)$ \\
\hline \multirow{6}{*}{ 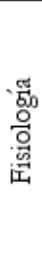 } & \multirow{2}{*}{$\begin{array}{l}\text { Producción } \\
\text { sustancias }\end{array}$} & EP & $4(5 \%)$ & & & & $14(17,5 \%)$ & $4(5 \%)$ & $1(1,3 \%)$ \\
\hline & & ESO & & $3(5,2 \%)$ & & & $9(15,5 \%)$ & $4(6,9 \%)$ & \\
\hline & \multirow{2}{*}{$\begin{array}{l}\text { Control de } \\
\text { temperatura }\end{array}$} & EP & $2(2,5 \%)$ & $4(5 \%)$ & & & & & \\
\hline & & ESO & & $4(6,9 \%)$ & & & & & \\
\hline & \multirow{2}{*}{ Otros } & EP & $8(10 \%)$ & & $2(2,5 \%)$ & $1(1,25 \%)$ & $17(21,3 \%)$ & $13(16,25 \%)$ & \\
\hline & & ESO & $10(17,2 \%)$ & $3(5,2 \%)$ & & $1(1,7 \%)$ & $9(15,5 \%)$ & $8(13,8 \%)$ & \\
\hline \multirow{8}{*}{ 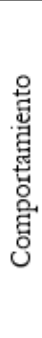 } & \multirow{2}{*}{ Socialización } & EP & & & & & $4(5 \%)$ & $2(2,5 \%)$ & $2(2,5 \%)$ \\
\hline & & ESO & & & & & $9(15,5 \%)$ & $10(17,2 \%)$ & $11(19 \%)$ \\
\hline & \multirow{2}{*}{ Cobijo } & EP & & & & & $3(3,8 \%)$ & $1(1,25 \%)$ & $1(1,3 \%)$ \\
\hline & & ESO & $2(3,4 \%)$ & $6(10,3 \%)$ & & & $4(6,9 \%)$ & & $3(5,2 \%)$ \\
\hline & \multirow{2}{*}{ Costumbres } & EP & $3(3,8 \%)$ & & & $1(1,25 \%)$ & $1(1,25 \%)$ & $2(2,5 \%)$ & $1(1,3 \%)$ \\
\hline & & ESO & & $6(10,3 \%)$ & & & $4(6,9 \%)$ & $3(5,2 \%)$ & $2(3,4 \%)$ \\
\hline & \multirow{2}{*}{ Alimentación } & EP & & & $1(1,25 \%)$ & & & $3(3,8 \%)$ & \\
\hline & & ESO & $3(5,2 \%)$ & & & & & $10(17,2 \%)$ & \\
\hline
\end{tabular}




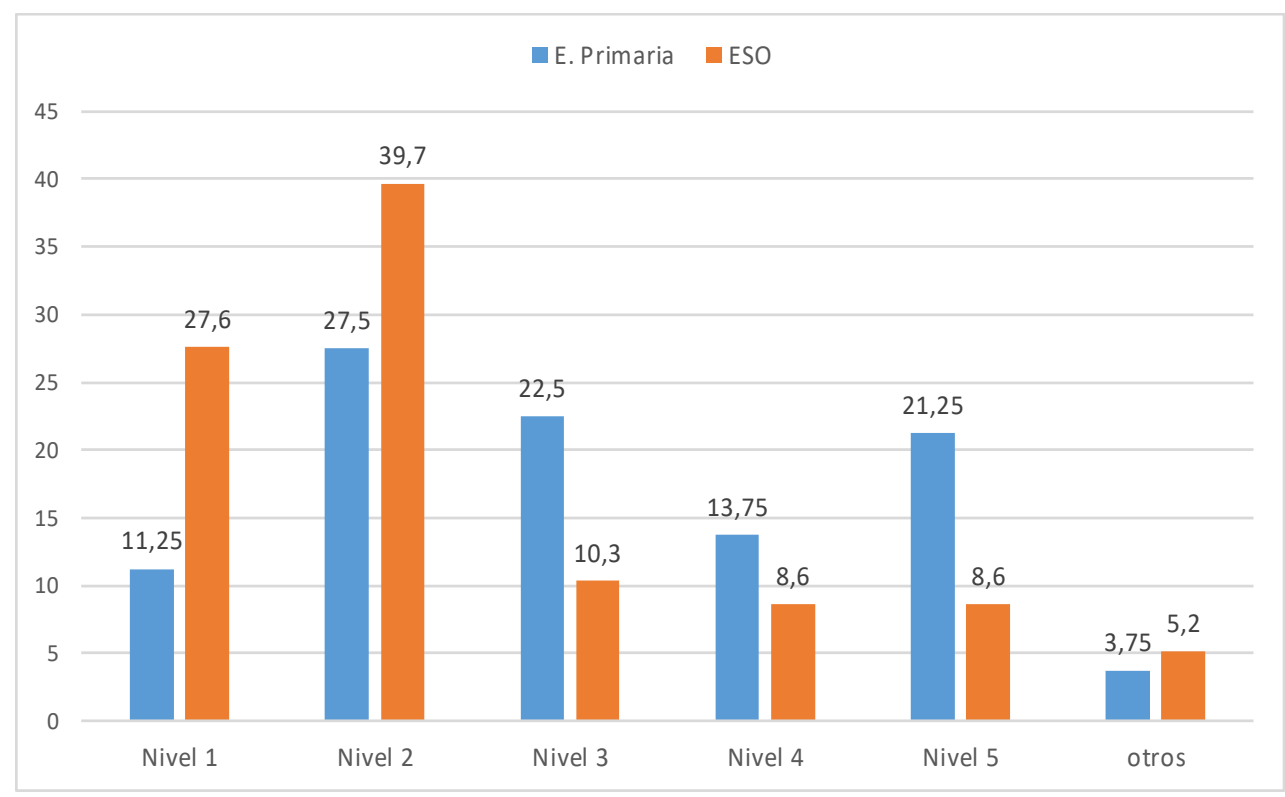

Figura 6. Porcentaje de diseños correspondientes a los distintos niveles de adecuación establecidos

\section{Conclusiones y discusión}

A partir del análisis realizado podemos concluir que:

1. Los estudiantes realizan una descripción prototípica del desierto, más adecuada y con un mayor nivel de detalle en el grupo de ESO.

2. Las adaptaciones de los animales se dirigen sobre todo a la obtención/conservación de agua y alimento y a la defensa de otros seres vivos, focalizándose éstas especialmente en rasgos morfológicos, seguidos de los fisiológicos y de comportamiento del animal, aunque se aprecian diferencias entre los dos colectivos -EP y ESO-.

3. En ambos grupos, las adaptaciones más consideradas en los animales diseñados, obtención de alimento y defensa, fueron las que se vincularon a una mayor diversidad de rasgos adaptativos.

Las mejores descripciones del desierto, realizadas por los estudiantes de ESO, y caracterizadas por la mayor consideración de especies, de adaptaciones al medio físico o de interacciones entre seres vivos, y la menor referencia a aspectos antropocéntricos, ponen de manifiesto una superior experiencia y madurez. Sin embargo, al igual que los estudiantes de EP caracterizan este medio sobre todo por la temperatura y el paisaje y en menor medida, a pesar de su importancia, por la escasez de precipitaciones, aspecto que coincide con otras experiencias realizadas con alumnos de EP (Horton y Friedenstab 2013).

Por otra parte, las adaptaciones más consideradas en los prototipos demuestran una especial preocupación por las necesidades primarias de los individuos (alimentación/protección). Concretamente la inquietud, por la obtención de agua y alimento pudo haber estado condicionada por la referencia explícita que a este particular se hace en la pregunta de la actividad. En cualquier caso, este tipo de adaptaciones fueron también prioritarias en otros estudios realizados con niños/as de menor edad (Frejd et al. 2020). Además, la preocupación por estas necesidades se ve reforzada por la diversidad de rasgos adaptativos asociados a las mismas encontradas en los diseños del alumnado. Sin embargo, la adaptación a otras características físicas del desierto, ampliamente nombrada en las descripciones del mismo, incluso por los alumnos de menor edad, como la temperatura, fueron menos consideradas, debido quizás a que la perciben como factor menos limitante para la vida, o a que les resulta 
difícil determinar el rasgo adaptativo específico. Precisamente, respecto a los rasgos adaptativos del animal, se puede admitir que los participantes pusieron en juego su imaginación, pues no solo consideraron características morfológicas típicas, sino que inventaron estructuras especiales y también atribuyeron a su animal capacidades diversas, como rapidez, astucia o inteligencia que lo hicieran más competitivo. Sin embargo, la consideración del comportamiento como foco de las adaptaciones resultó muy poco frecuente en EP, poniendo de manifiesto la dificultad de los niños para visualizar interacciones, especialmente dentro de una misma población, para conseguir alimento, pareja, etc., aspecto que resulta coherente con la dificultad de captar el concepto de población (Jiménez-Tejada, Sánchez-Monsalve y González-García 2013).

En términos generales, las adaptaciones que emergen de los diseños realizados constituyen una forma de evidenciar el modelo de ser vivo que subyace en la mente de sus autores (Gil Quilez et al. 2011) y su diferencia respecto a la edad. En este sentido, y aunque ambos grupos revelan una preocupación por las adaptaciones a las necesidades primarias, en ESO se añaden en mayor medida adaptaciones reproductivas (buscar pareja, cuidado de crías). Además, y aunque los estudiantes han vinculado las adaptaciones más consideradas a un amplio abanico de rasgos de su animal, dichos rasgos también se amplían de manera evidente entre los estudiantes de mayor edad, de tal forma que sus prototipos responden a un mejor nivel de adecuación.

Cabe señalar que las ideas que han activado niños y adolescentes respecto al desierto y a la adaptación, nos sirven de base para plantear recomendaciones didácticas concretas. En primer lugar, sería conveniente ampliar la visión de desierto prototípico habitual, aludiendo a otros, por ejemplo, al desierto helado. También sería necesario insistir en las adaptaciones asociadas al comportamiento, por ser las menos consideradas. Para ello resulta útil favorecer la visualización de la población, ofreciendo ejemplos de gregarismo (manadas, bancos de peces...) por ser una oportunidad para identificar adaptaciones diversas no solo asociadas a los factores bióticos del medio (diferentes interacciones interespecíficas como el mutualismo e intraespecíficas como la colaboración para obtener alimentos), sino también vinculadas a los factores abióticos (protección frente a las inclemencias del tiempo, como por ejemplo el comportamiento de los pingüinos ante las tormentas de nieve). Todo ello ayuda a ir captando la idea de población.

Sin embargo, a pesar de su importancia, el tratamiento de la adaptación encierra problemas, pues puede contribuir a la promoción de ideas simplistas y deterministas, caracterizadas por la concepción de que la evolución -selección natural- conduce a la perfecta adaptación de todos los órganos del individuo. Este aspecto se solventaría admitiendo la falta de conocimiento existente sobre la función de ciertas estructuras (por ejemplo, las muelas de juicio...) o relativizando las ventajas de los rasgos adaptativos de especies concretas. Sirva de ejemplo en este último caso: la altura de las jirafas que constituye una desventaja para que el animal beba; el bipedismo y el tamaño de la pelvis que dificulta el parto en nuestra especie, etc. Otro punto oscuro del estudio de la adaptación podría ser la posibilidad de potenciar explicaciones lamarkistas, sin embargo, esta explicación puede concebirse como un paso intuitivo, ampliamente descrito, que habrá que hacer evolucionar en un proceso de modelización, siendo el contraste de modelos una posible vía de aprendizaje (Oliva 2019). De todas formas, la construcción de un modelo abstracto debería pasar por un escenario, caracterizado por la diversidad y la adaptación, en el que surjan las preguntas mediadoras (Márquez y Roca 2006).

Independientemente de las dificultades citadas, la adaptación se puede tratar en los primeros niveles educativos. A nuestro juicio, mostrar a los niños de EP la variedad de rasgos adaptativos en relación a diversos factores, incluyendo los no considerados inicialmente, y 
presentarles la variedad de soluciones que aportan los órganos análogos, ayudará a percibir la diversidad de seres vivos en interacción con su medio. De esta forma se contribuye a organizar el conocimiento biológico desde una perspectiva ecológica y evolutiva (Bermudez y Occelli 2020), donde la diversidad constituye una de las grandes ideas a considerar en la construcción del modelo evolutivo (Fuentes Silveira y García Barros, 2015; Lehrer y Schauble 2012), aunque no se trate en «estricto sensu» la evolución. Sin embargo, desafortunadamente, el curriculum LOMCE mantiene una visión de la diversidad excesivamente descriptiva, omitiendo la adaptación como contenido en EP, dificultando así la promoción de las primeras explicaciones del funcionamiento de la vida en interacción. La insistencia en la diversidad descriptiva también se traslada a $1^{\circ}$ de ESO, donde la adaptación se desvincula del contenido explicativo que ofrece los ecosistemas. Por ello urge la superación de estas tradiciones en la escuela.

Cabe indicar que, aun siendo conscientes de que estamos haciendo un reduccionismo del concepto de adaptación al considerar solo su sentido evolutivo, omitiendo las adaptaciones fisiológicas, no hereditarias, que responden a estímulos resultando más eficaces las funciones de los sistemas (Prevosti 1997), consideramos trascendente que los estudiantes, no solo reflexionen sobre las necesidades adaptativas, sino también que capten el dinamismo del medio y su impacto en las nuevas adaptaciones de las poblaciones, en función de su diversidad intraespecífica y de su tamaño. Este tipo de reflexión es recomendable realizarlo al final de EP y de $1^{\circ}$ de ESO, pues se van sentado las bases de la comprensión del proceso evolutivo a través de sucesivas preguntas que apuntan a una secuencia de ideas clave cada vez más complejas y abstractas: hay diversidad de medios y de adaptaciones, pero si el medio cambia, ¿quién o quiénes sobrevivirán?, ¿es importante que exista variedad de individuos dentro de una población?, entonces, ¿cuál es el mecanismo de la selección y la supervivencia?, ¿cómo podemos explicar la existencia de la variabilidad en las poblaciones, qué papel juega la reproducción sexual?, ¿cómo podemos explicarla desde la perspectiva genético/molecular?, etc. De este modo el escenario o situación presenta los fenómenos biológicos y los modelos funcionales y evolutivos los explican, ambos son trascendentes en el aprendizaje, pues no se puede explicar una realidad que no se conoce y que no se siente la necesidad de hacerlo. Lo indicado también enriquecerá el modelo de ser vivo, pues la capacidad de adaptación y cambio es poco considerada como atributos inherentes a la vida por el alumnado de distintos niveles (García-Barros, Fuentes Silveira y Ramos Fariña, 2015).

\section{Agradecimientos}

Agradecemos la colaboración inestimable del alumnado y profesorado de los centros participantes en este estudio. Este se enmarca en el proyecto EDU2016-79563-R (FEDER/Ministerio de Economía Industria y Competitividad y AGI)

\section{Referencias}

Acher A. (2014) Cómo facilitar la modelización científica en el aula. Tecné, Episteme y Didaxis 36, 63-75. http://dx.doi.org/10.17227/01213814.36ted63.75

Araújo L. A. L. (2020) The centrality of evolution in biology teaching: towards a pluralistic perspective. Journal of Biological Education 1-12. https://doi.org/10.1080/00219266.2020.1757486

Bermudez G.M.A., Occelli M. (2020) Enfoques para la enseñanza de la Biología: una mirada para los contenidos. Didáctica de las Ciencias Experimentales y Sociales 39, 135-148. http://doi.org/10.7203/DCES.39.16854 
Borgerding L. A., Kaya F. (2019) Preschool children's ideas about biological adaptation during a science camp. International. Journal of Science Education 41 (17), 2410-2429. http:// doi.org/10.1080/09500693.2019.1683911

Cañal P. (2004) Las plantas, ¿fabrican sus propios alimentos? Hacia un modelo escolar alternativo sobre la nutrición de las plantas. Alambique 42, 55-71.

Cañal P. (2008) Investigando los seres vivos. Proyecto Curicular Investigando Nuestro Mundo (6-12). Sevilla: Diada.

Caravita S., Falchetti E. (2005) Are bones alive? Journal of Biological Education 39 (4), 163-170.

Clement J. J. (2000) Model based learning as a key research area for science education. International Journal of Science Education 22(9), 1041-1053. https://doi.org/10.1080/095006900416901

COSCE-Confederación de Sociedades Científicas de España (2011) Informe ENCIENDE. Enseñanza de las Ciencias en la Didáctica Escolar para edades tempranas en España. Madrid: COSCE. http://www.cosce.org/disponible-el-informe-enciende/

De la Gándara M., Gil-Quílez M. J., Sanmartí N. (2002) Del modelo científico de «adaptación Biológica» al modelo de "adaptación Biológica» en los libros de texto de enseñanza secundaria obligatoria. Enseñanza de las Ciencias 20 (2), 303-314.

Frejd J., Stolpe K., Hultén M., Schönborn K. J. (2020) Making a fictitious animal: 6-7 year-old Swedish children's meaning making about evolution during a modelling task. Journal of Biological Education, 1-17. https://doi.org/10.1080/00219266.2020.1799843

Fuentes Silveira, M. J., García Barros, S. (2015). El estudio de la biodiversidad. Una propuesta de progresión para primaria y secundaria obligatoria. Alambique, 79, 25-34.

Garbisu C., Amézaga I., Albizu I., Alkorta I. (2003). La esencia de los seres vivos. Ecosistemas 12 (3). https://doi.org/10.7818/ECOS.355

García Barros S, Fuentes Silveira M.J., Ramos Fariña, P. (julio 2018) Ideas about the model of what a living is being expressed by secondary school pupils and teachers in training. [Presentación de comunicación] XII Conference of European Research in Didactics of Biology -ERIDOB-, Zaragoza, España.

García Rovira M.P. (2005) Los modelos como organizadores del currículo en biología. Enseñanza de las Ciencias n ${ }^{\circ}$ extra, 1-6.

Garrido M., Martínez Losada C. (2009) ¿Qué enseñar sobre los seres vivos? Aula de innovación educativa 183-184, 34-36.

Gil Quílez M. J., de La Gándara Gómez M., Dies Álvarez M. E., Martínez Peña B. (2011) Animales extraordinarios: la construcción y uso de modelos en la Escuela Primaria. Investigación En La Escuela (74), 89-100. https://doi.org/10.12795/IE.2011.i74.07

Gould S.J., Lewontin R.C. (1979) The spandrels of San Marco and the Panglossian paradigm: A critique of the adaptationist programme. Proceedings of the Royal Society London B 205 (1161), 581-598. https:// doi.org/10.1098/rspb.1979.0086

Harlen W. (2015) Trabajando con las grandes ideas de la educación en ciencias. Trieste, Italia: Programa de Educación en Ciencias (SEP) de la Red Global de Academias de Ciencias (IAP). tinyurl.com/rqifqky3

Horton J., Friedenstab S. (2013) Desert Survivors! Science and Children 51 (1), 59-65. 
Jiménez-Tejada M. P., Sánchez-Monsalve C., González-García F. (2013) How Spanish primary school students interpret the concepts of population and species. Journal of Biological Education 47 (4), 232-239.

Kevin J. Pugh, Kristin L.K. Koskey, Linnenbrink-Garcia, L. (2014) High school biology students' transfer of the concept of natural selection: a mixed-methods approach. Journal of Biological Education $48 \quad$ (1), 23-33. https://doi.org/10.1080/00219266.2013.801873

Larrain A., López P., Gómez M., Verdugo S., Freire P., Grau V., Cosmelli D., Bonhomme A. (2021) Las ideas de estudiantes chilenos acerca de la evolución de los seres vivos: ¿qué piensan al término de la enseñanza primaria? Revista Eureka sobre Enseñanza y Divulgación de las Ciencias 18 (1), 1106-1- 1106-20. https://doi.org/10.25267/Rev_Eureka_ensen_divulg_cienc.2021.v18.11.1106

Lehrer R., Schauble L. (2012) Seeding evolutionary thinking by engaging children in modelling its foundations. Science Education 96 (4), 701-724. https://doi.org/10.1002/sce.20475

Márquez C., Roca M. (2006) Plantear preguntas: un punto de partida para aprender ciencias. Revista Educación y Pedagogía 18 (45), 61-71.

Martínez Losada, C., García Barros, S., Garrido, M. (2014) How children characterise living beings and the activities in which they engage. Journal of Biological Education, 48(4), 201210. https://doi.org/10.1080/00219266.2013.849281

Maturana H., Varela F. J. (1981) Autopoiesis and Cognition: The 60 Realization of the Living. Boston: Dordrecht, D. Reidel.

Moreno J. (2006) Ecología: su importancia para el estudio de la evolución. Evolución 1 (1), 2542.

NRC (2007) Taking Science to School.: Learning and Teaching Science in Grades K-8. Washington, DC: The National Academic Press.

Oliva J.M. (2019) Distintas acepciones para la idea de modelización en la enseñanza de las ciencias. Enseñanza de las Ciencias 39 (2), 6-24. https://doi.org/10.5565/rev/ensciencias.2648

Pérez G., Gómez Galindo A.A., González Galli L. (2018) Enseñanza de la evolución: fundamentos para el diseño de una propuesta didáctica basada en la modelización y la metacognición sobre los obstáculos epistemológicos. Revista Eureka sobre Enseñanza y Divulgación de las Ciencias 15(2), 2102-1-2102-1-13. https://doi.org/10.25267/Rev_Eureka_ensen_divulg_cienc.2018.v15.i2.2102

Prevosti A. (1997) La adaptación en biología. Alambique 11, 93-101.

Prinou L., Halkia L., Skordoulis C. (2011) The Inability of Primary School to Introduce Children to the Theory of Biological Evolution. Evolution: Education and Outreach 4 (2), 275-285. https://doi.org/10.1007/s12052-011-0323-8

Pujol R. M. (2003) Didáctica de las Ciencias en la Educación Primaria. Madrid: Síntesis.

Reeve H. K., Sherman P. W. (1993) Adaptation and the goals of evolutionary research. The Quarterly Review of Biology 68(1), 1-32.

Rowlands O., Hokayem H., Biediger D. (2017) The case of polar bears. Science and Children 55 (3), 60-65.

Royce C. A. (2017) The Tool and Eye. Science and Children 55 (1), 22-27. 
Royce C. A. (2019) Looking Good: Structural Adaptations and Inherited Traits. Science and Children 57 (4), 18-25.

Rule A.C., Baldwin S., Schell R. (2008) Second Graders Learn Animal Adaptations through Form and Function Analogy Object Boxes. International Journal of Science Education 30 (9), 1159-1182. https:// doi.org/10.1080/09500690701385266

Sanmartí N., Márquez C. (2017) Aprendizaje de las ciencias basado en proyectos: del contexto a la acción. Ápice. Revista de Educación Científica 1(1), 3-16. https://doi.org/10.17979/arec.2017.1.1.2020

Vázquez-Ben L. y Bugallo-Rodríguez A. (2018) El modelo de evolución en el curriculum deEducación Primaria: Un análisis comparativo en distintos países. Revista Eureka sobre Enseñanza y Divulgación de las Ciencias15 (3), 3101-1-3101-13. doi: 10.25267/Rev_Eureka_ensen_divulg_cienc.2018.v15.i3.3101 
Anexo 1. Ejemplos de dibujos, desierto/animales

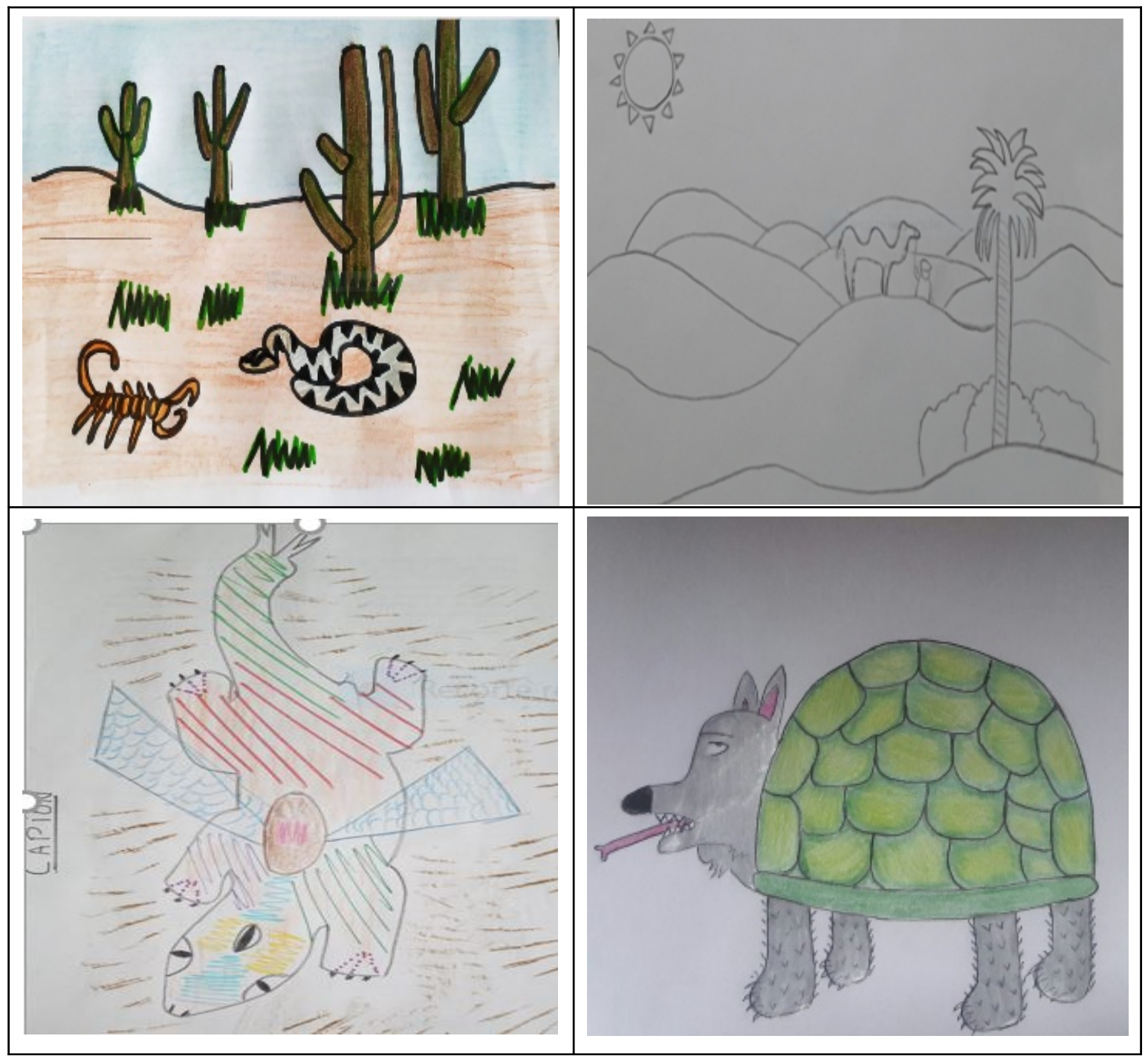

